\title{
Isolated sarcoidosis of accessory spleen in the greater omentum: A case report
}

\author{
CHAOYONG TU ${ }^{1}$, QIAOMEI LIN ${ }^{1}$, JINGDE ZHU ${ }^{1}$, CHUXIAO SHAO $^{1}$, KUN ZHANG $^{1}$, CHUAN JIANG $^{1}$, \\ ZHIYONG DING ${ }^{1}$, XINGMU ZHOU ${ }^{2}$, JIEFEI TU ${ }^{1}$, WANLIN ZHU ${ }^{1}$ and WEI CHEN ${ }^{3}$ \\ Departments of ${ }^{1}$ General Surgery and ${ }^{2}$ Pathology, Lishui Central Hospital, Lishui, Zhejiang 323000; \\ ${ }^{3}$ Department of Hepatobiliary and Pancreatic Surgery, Second Affiliated Hospital, \\ Zhejiang University School of Medicine, Hangzhou, Zhejiang 310009, P.R. China
}

Received January 16, 2015; Accepted February 19, 2016

DOI: $10.3892 /$ etm.2016.3221

\begin{abstract}
Sarcoidosis is a multisystemic disease of unknown origin characterized by the formation of non-caseating granulomas. Thoracic involvement is the most common presentation; however, sarcoidosis can involve almost any other organ. To the best of our knowledge there have been only 10 cases of splenic sarcoidosis reported in the English literature, with no reports of sarcoidosis of an accessory spleen. The present study reports a case of isolated sarcoidosis of an accessory spleen in the greater omentum, which was identified postoperatively in a 44-year-old female. Chest X-ray results were normal. Gastric endoscopy demonstrated an ulcer in the antrum, which was confirmed to be a signet-ring cell carcinoma via biopsy. Computed tomography of the abdomen revealed mild thickening of the posterior antrum, and a mass in the inferior pole of the left kidney. Intraoperatively, no masses were detected in the liver and spleen. Moreover, no enlarged lymph nodes were detected in the abdominal cavity, pelvic cavity, mesenteric and para-aorta. Following a radical distal gastrectomy and left radical nephrectomy, postoperative pathology demonstrated signet-ring cell carcinoma in the antrum, left renal clear cell cancer and a red lesion measuring $0.5 \times 0.5 \mathrm{~cm}$ in the greater omentum, which was similar to the spleen in the splenic cavity and was regarded as an accessory spleen. Following exclusion of fungi and acid-fast bacilli as causative agents, sarcoidosis of the accessory spleen in the greater omentum was confirmed. The patient recovered uneventfully and was discharged on day 8 postoperation. The patient remained alive after two-year follow-up without sarcoidosis and malignant tumor recurrence. The present case demonstrated that, intraoperatively,
\end{abstract}

Correspondence to: Dr Wei Chen, Department of Hepatobiliary and Pancreatic Surgery, Second Affiliated Hospital, Zhejiang University School of Medicine, 88 Jiefang Road, Hangzhou, Zhejiang 310009, P.R. China

E-mail: viogro@163.com

Key words: sarcoidosis, accessory spleen comprehensive exploration should be conducted to exclude the accessory spleen, which may also suffer from sarcoidosis.

\section{Introduction}

Sarcoidosis is a multisystemic disease of unknown origin characterized by the formation of non-caseating granulomas. Sarcoidosis occurs worldwide and should be diagnosed upon the clinical presentation for non-caseating granulomas, which are also associated with other infectious diseases, autoimmune disorders and neoplasia. Sarcoidosis is often asymptomatic (1); however, its symptoms may be systemic such as fatigue, fever or weight loss, or organ-specific, such as shortness for breath or cough. Thoracic involvement is the most common presentation, reported in up to $90 \%$ of patients (2). However, sarcoidosis can involve almost any other organ, including the skin, eyes and abdominal organs (2). Extra-thoracic disease can occur in association with or in the absence of intra-thoracic disease (3). Involvement of abdominal viscera is seldom, which may resemble certain common infectious or neoplastic conditions. Involvement of the liver and spleen is observed in $40-60 \%$ of autopsies $(4,5)$. However, an isolated extrathoracic sarcoidosis accounts for only $10 \%$ of patients (6). To the best of our knowledge there have been only 10 cases of splenic sarcoidosis reported in the English literature, data from which are summarized in Table I. Accessory spleens (AS) might be formed during embryonic development as separated or ectopic splenic tissue. They are usually near the splenic hilum, but may be located in the greater omentum (7). There are no previous reports of accessory splenic sarcoidosis. Herein, we report a case of isolated sarcoidosis of an accessory spleen in the greater omentum, which was identified postoperatively in a 44-year-old female.

\section{Case report}

A 44-year-old female presented to the Department of General Surgery of Lishui Central Hospital (Lishui, China) complaining of pain beneath the xiphoid process for two years. The patient denied any personal history of surgery or family history of cancer. Physical examination presented with normal results. The liver and spleen presented normally. Chest X-ray results 
were normal (Fig. 1). Gastric endoscopy showed an ulcer in the antrum measuring $1.5 \times 1.5 \mathrm{~cm}$, which was confirmed to be a signet-ring cell carcinoma by biopsy. Computed tomography (CT) of the abdomen revealed mild thickening of the posterior antrum, and a mass measuring $5 \times 4 \mathrm{~cm}$ in the inferior pole of the left kidney. No abnormal foci could be found in the greater omentum or other organs. There was no associated splenomegaly (Figs. 2 and 3). Mycobacterial, fungal, bacterial and parasitic infections were all excluded. After four days of preoperative preparation, including liver function, renal function and blood coagulation function blood tests and the exclusion of surgical contraindications, the patient underwent surgical exploration. Intraoperatively, a solid ulcer was detected in the antrum measuring $1 \times 1 \mathrm{~cm}$, and a solid mass measuring $5 \times 4 \mathrm{~cm}$ was identified in the inferior pole of left kidney. The accessory spleen in the greater omentum was not explored. There were no evident masses in the liver and spleen. In the abdominal cavity, pelvic cavity, mesenteric and para-aorta, no enlarged lymph nodes were detected. Radical distal gastrectomy and left radical nephrectomy, with a midline incision from the xiphoid to umbilicus, were performed.

Radical distal gastrectomy was completed as follows: i) The greater omentum was separated from the transverse colon, followed by resection of the superior leaf of the transverse mesocolon; ii) the gastroepiploic vein and artery were separately ligated, and the anterior pancreatoduodenal and infrapyloric nodes, retroduodenopancreatic lymph nodes and nodes of inferior portion of the hepatododenal ligament were resected; iii) the right gastric artery was ligated, and the duodenum was transected and closed $\sim 3 \mathrm{~cm}$ from the pylorus; iv) the lymph nodes and the tissue surrounding the celiac trunk were resected, and the left gastric vein was ligated at its origin followed by the left artery; v) dissection continued up the lesser curvature of the stomach, where the right paracardial zone was dissected and two short gastric veins were ligated; vi) the upper portion of the stomach was transected using a Proximate linear cutter (Ethicon Endo-Surgery, LLC, Guaynabo, Puerto Rico), and the right broken line of the stomach was $3 \mathrm{~cm}$ below the cardia, whereas the left was above the origin of the second resected gastric short vein; and vii) Hofmeister's gastrojejunal anastomosis (Billroth II) was performed (8). Left radical nephrectomy was performed as follows: i) Left posterior peritoneum and perirenal fascia were incised; ii) after the left renal pedicle was divided, the left renal artery and vein were separately ligated; iii) the left renal, perirenal fat and fascia were divided; and iv) after the left ureter was resected below the iliac vessels, the specimen was removed.

In addition to signet-ring cell carcinoma in the antrum and the left renal clear cell cancer, postoperative pathology demonstrated a red lesion measuring $0.5 \times 0.5 \mathrm{~cm}$ in the greater omentum, which was similar to the spleen in the normal splenic cavity and was thus regarded as an accessory spleen. Histological examination of the lesion in the greater omentum revealed small and dense epithelioid non-caseating granulomas, with clear margins surrounded by a small quantity of lymphocytic infiltrates (Fig. 4). Fungi were excluded using periodic acid-Schiff and hexamine silver staining. Acid-fast bacilli were excluded by acid-fast staining. Acid-Schiff and hexamine silver staining were performed using $0.5 \%$ potassium permanganate solution, $8 \%$ chromic acid solution, $5 \%$ silver nitrate solution, $3 \%$ hexamethylene tetramine solution, $5 \%$ sodium tetraborate solution, $0.1 \%$ gold chloride solution and 5\% sodium thiosulfate (Sinopharm Chemical Reagent Co., Ltd., Shanghai, China). Phenol fuchsine fluid and Mayer's hemalum solution (Fuzhou Maixin Biotech. Co., Ltd., Fuzhou, China) were used for acid-fast bacilli staining.

In consideration of the aforementioned tests, sarcoidosis of accessory spleen in the greater omentum was confirmed. The patient recovered uneventfully and was discharged on day 8 postoperation. The patient remained alive after two-year follow-up without sarcoidosis and malignant tumor recurrence.

\section{Discussion}

In the present study a rare case of sarcoidosis of accessory spleen in the greater omentum was reported. Sarcoidosis is observed worldwide, the incidence rate of the disease, organs involved, manifestations and prognosis vary widely with geography and ethnicity. A diagnosis of sarcoidosis is based on clinical and radiological results (6), in addition to the histology of epithelioid granulomas (9). However, granulomas are not a specific finding of sarcoidosis, and other diseases such as infections caused by bacteria or fungi and environmental agents must be excluded. The lungs are involved in $90 \%$ of patients with sarcoidosis; however, almost any other organ may be involved, yet the digestive tract is rarely involved $(2,5,10,11)$. Gastrointestinal symptoms might exist and are variable depending on the location of disease $(8,12,13)$. The frequency of splenomegaly in sarcoidosis may range between 1 and $40 \%(14,15)$. Correlation between pulmonary abnormalities and hepatosplenic involvement has not yet been established (16). Autopsy studies have revealed splenic involvement in 38-77\% of patients with sarcoidosis (1). Isolated splenic sarcoidosis is rare (1). Sarcoidosis of the spleen without clinical or radiographic pulmonary disease is exceedingly rare, with only sporadic cases reported. There have been only 10 cases of isolated splenic sarcoidosis, and no reports of sarcoidosis in the accessory spleen (Table I). Due to the popularity and small size of the accessory spleen, the operator ignored it intraoperatively. Splenic sarcoidosis exhibits a female preference ( $7: 3$ female to male ratio), with a median age of 46.5 years. Patients may be asymptomatic $(9,17)$, or exhibit abdominal pain $(12,16)$. Other atypical symptoms include sweats $(18,19)$, weakness and weight loss $(20)$. The incidence of splenomegaly associated with sarcoidosis is variable, but may be has high as $40 \%$ (21). Massive splenomegaly is quite rare; Fordice et al (22) reviewed 6,074 cases of sarcoidosis, 628 patients had quantified splenomegaly and $20(3 \%)$ had massive splenomegaly. Splenic infarction due to sarcoidosis was has been reported (23). Symptoms related to hypersplenism with anemia, thrombocytopenia and leucopenia have been reported (6). However, massive splenomegaly in sarcoidosis may not result in a pathophysiological effect (17). As the present case exhibited two malignant tumors. Whether the incidence of splenic sarcoidosis is related to the tumors requires confirmation.

Routine laboratory tests are not usually helpful in the diagnosis of sarcoidosis as these tests are nonspecific. However, peripheral lymphopenia with CD4 depletion, elevated levels of serum-angiotensin converting enzyme (S-ACE), lysozyme, 


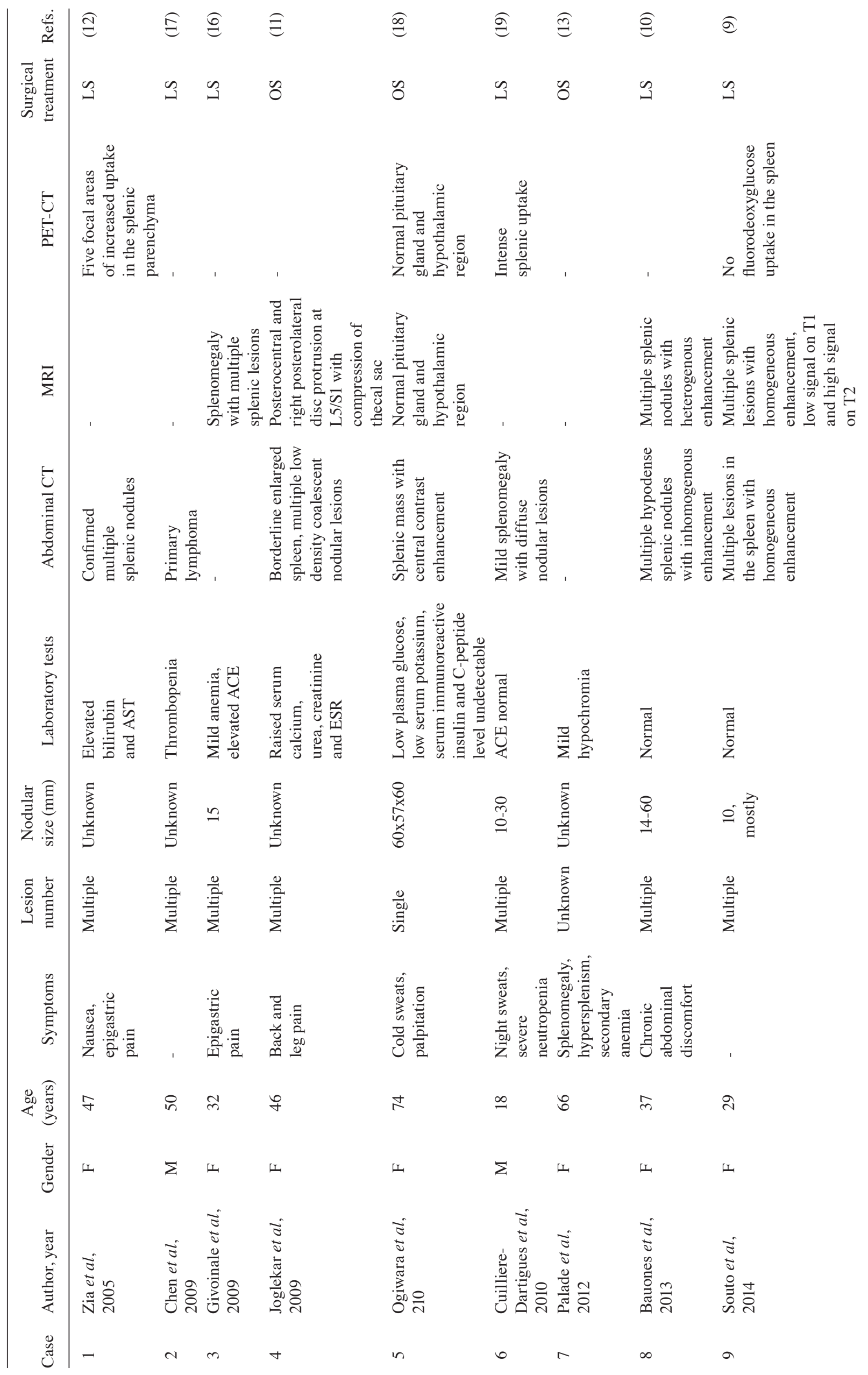




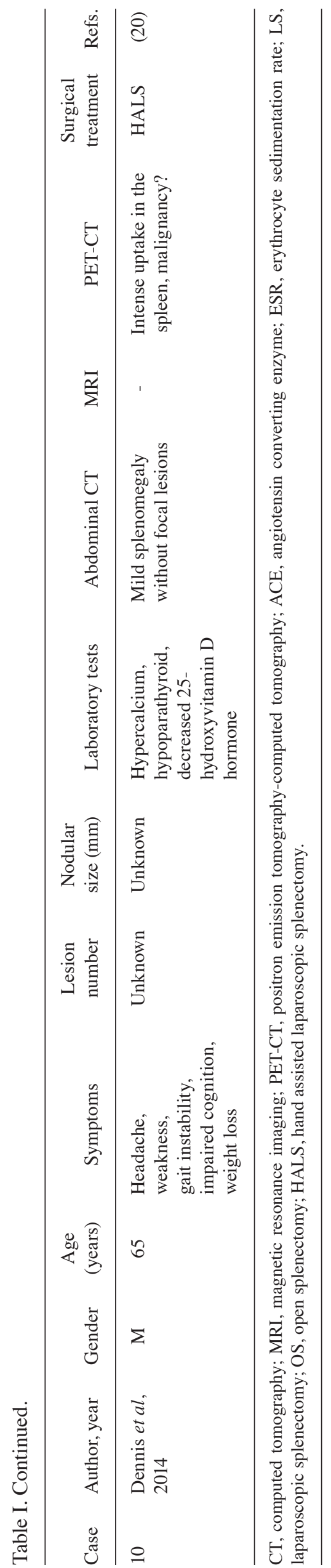

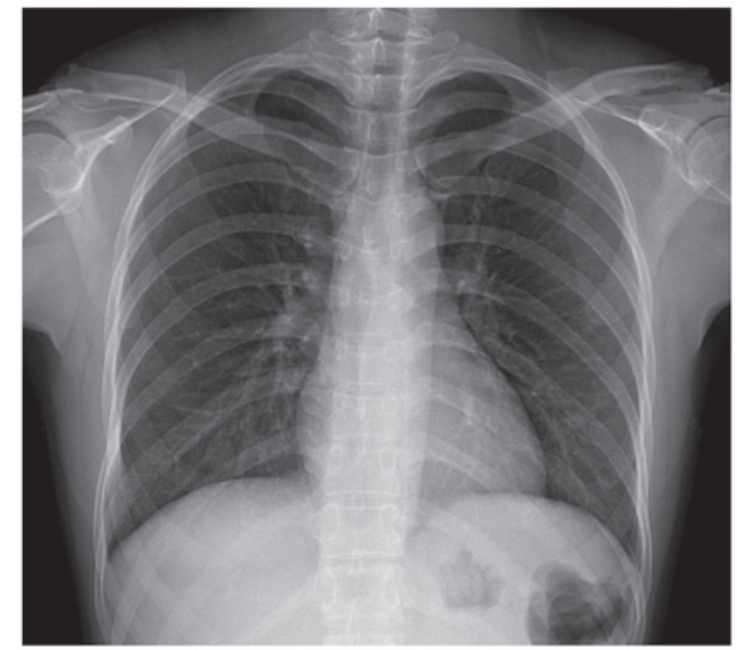

Figure 1. Chest X-ray showing no foci in the lungs.

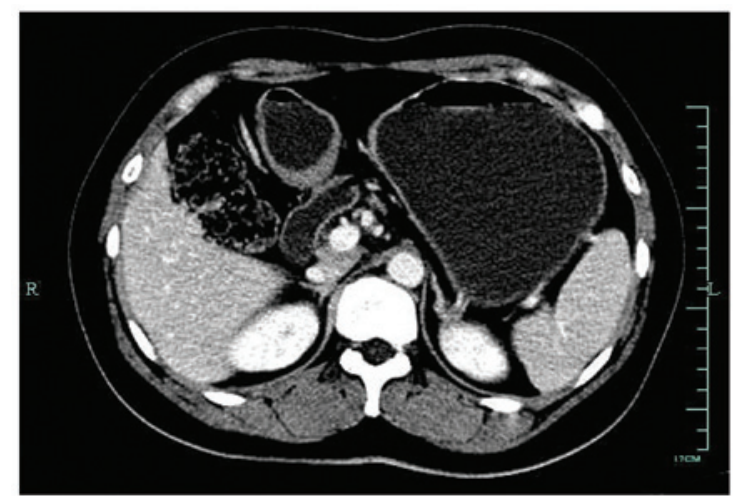

Figure 2. Contrast computed tomography showing mild thickening of the posterior antrum and normal spleen.

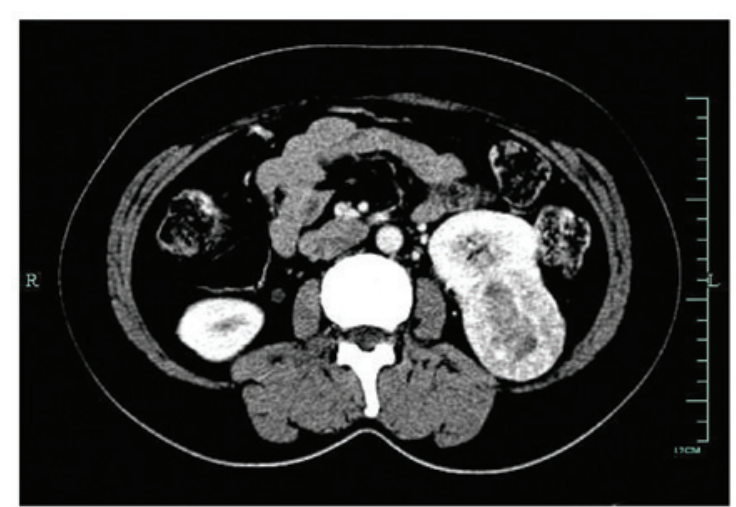

Figure 3. Contrast computed tomography showing a mass measuring $5 \times 4 \mathrm{~cm}$ in the inferior pole of left kidney.

$\beta-2$ microglobulin, hypercalcemia and hypercalciuria can be helpful to the diagnosis (24). ACE (25) is elevated in $60 \%$ of cases, hypercalcemia or hypercalciuria occur in about $10-40 \%$ of patients (6). Lymphocytic alveolitis with a high CD4/CD8 ratio in bronchoalveolar lavage (BAL) fluid is highly suggestive of the disease (24). Warshauer et al (1) observed a linear association between serum ACE level and spleen size as well as between ACE level and the presence of hepatosplenic 


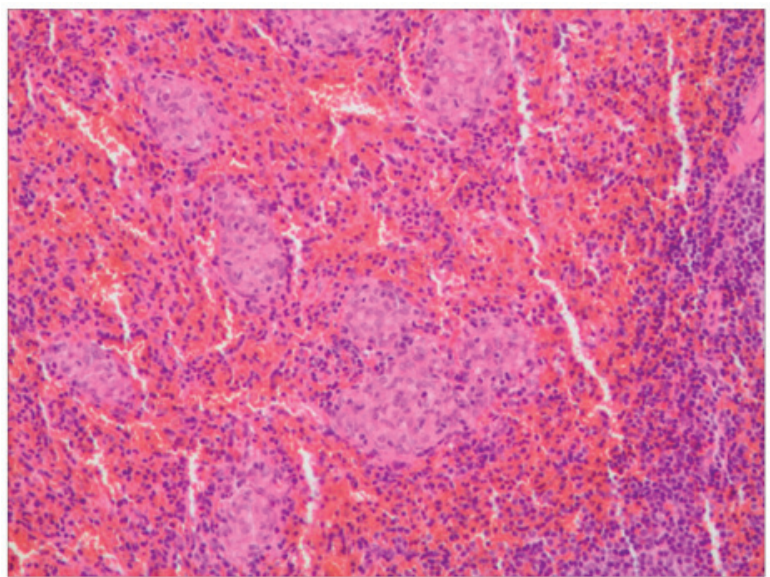

Figure 4. Small and dense epithelioid non-caseating granulomas with clear margin surrounded by a small quantity of lymphocytic infiltration (stain, hematoxylin and eosin; magnification, x100).

nodules; however, no correlation between the degree of chest abnormality and the involvement of liver and spleen was identified (2). In these cases, the diagnosis of sarcoidosis could be difficult as sarcoidosis is often not considered due to the nonspecific nature of laboratory tests.

The imaging features of splenic sarcoidosis are variable. Radiographic results of isolated splenic lesions are nonspecific and own many differential diagnoses including lymphoma, metastatic disease, hematomas, abscesses, hamartomas, and angiosarcomas (26). Certainly, a histopathologic diagnosis for definitive diagnosis is needed. The spleen is often enlarged; and hypodense splenic nodules can be seen in approximately $15 \%$ of patients (1). Lesions are often multiple and diffused distributed; however, they may be undetectable (20). The majority of nodules are between 0.1 and $3.0 \mathrm{~cm}$, with a mean of $\sim 1.0 \mathrm{~cm}$. Notably, Bauones et al (10) observed capsular retraction on ultrasound and magnetic resonance images (MRI), and confirmed at gross examination. This unreported imaging feature in splenic sarcoidosis may be associated with the presence of significant collagen fibrosis that surrounded the granulomas. Notably, in the spleen capsular retraction is rarely reported, in relation to splenic infarction or neoplastic disease (27). In particular, there was no thoracic and lymphatic system involvement to suggest sarcoidosis in this patient. Kessler et al (28) concluded that focal lesions in spleen in sarcoidosis patients can occasionally be detected by ultrasound; however, diffuse increased homogeneous or heterogeneous echogenicity is more common. Pérez-Grueso et al (5) initially adopted contrast enhanced ultrasound (CEUS) to diagnose splenic sarcoidosis. Grzelak et al (29) implemented CEUS to diagnose two cases of hepatosplenic sarcoidosis, and concluded that CEUS has the potential to become a reliable tool; replacing more expensive and less available imaging methods, such as CT and MRI. However, certain cases exhibited a tumor-like pattern under CEUS; consisting of slight but diffuse enhancement of the lesions in the arterial phase followed by progressive hypoenhancement in the parenchymal phase (30). Tana et al (31) speculated that this may be related to different vascularization of the lesions and suggested the need for further studies before making definite conclusions. In one report, lesions visible on contrast-enhanced CT were not observed under sonography, suggesting that the acoustic impedance of the granulomas was similar to that of normal splenic tissue $(11,32)$. Dynamic gadolinium-enhanced MRI is useful in characterizing splenic lesions and narrowing the differential diagnosis. Classically sarcoidosis-related splenic lesions are T1 and T2 hypointense and relatively hypoenhancing (33). Positron emission tomography-CT is a useful imaging method for the evaluation of disease activity and the identification of areas that are undetectable by ultrasound, CT and MRI, as well as in monitoring response to treatment in patients with sarcoidosis. Among the reviewed cases, which employed PET-CT, intense fluorodeoxyglucose uptake in the spleen was detected in $75 \%$ of patients $(9,12,19,20)$. Kataoka et al (34) suggested multiple imaging modalities, including dynamic CT and MRI for detecting splenic lesions of sarcoidosis. Due to the risk of bleeding, tract seeding and variable results, a percutaneous biopsy via ultrasound or CT was not suggested. Without lung or other systemic involvement, the symptoms of splenic sarcoidosis may resemble more serious neoplastic or infectious disease (6), and in this context only splenectomy allows the establishment of a diagnosis. Following diagnosis, patients require continual follow-up for systemic manifestations and associated complications of sarcoidosis. None of the presently reviewed 10 cases were diagnosed preoperatively. The present case was accidently identified postoperative pathology.

Splenectomy is considered as a treatment option for hypersplenism, prophylaxis for splenic rupture and neoplastic exclusion (16). Splenectomy might not alter the course of disease and is merely an adjunct to medical therapy in order to resolve complications of sarcoidosis (35). With the improvements in the technologies, laparoscopic splenectomy has gradually become the gold standard for surgical removal of the spleen, and there have been reported laparoscopic splenectomies performed for sarcoidosis $(9,12)$. Laparoscopic splenectomy is a minimally invasive procedure that provides superior aesthetic results, decreased blood loss and decreased postoperative complications and faster recovery, which allows the earlier initiation of chemotherapy. If a splenectomy is to be performed, comprehensive exploration should be conducted to exclude the accessory spleen, which may also suffer from sarcoidosis. In the presently reviewed cases, hypercalcemia and hypersplenism disappeared after splenectomy $(11,13)$. As isolated splenic disease may be only a precursor to future systemic disease, and thus patients must be followed over their lifetimes. Asymptomatic patients do not require the immunosuppressive drugs; however, if symptoms do occur, steroids or even methotrexate and azathioprine can be used. As many as $66 \%$ of patients spontaneously remit (6). The lack of response or the appearance of complications may require splenectomy (12). In the majority of cases the recommended therapy is using glucocorticoid, which should be resumed in the instance of a relapse.

In summary, one rare case of isolated sarcoidosis of accessory spleen in the greater omentum is reported. Symptoms of splenic sarcoidosis are atypical, requiring multiple imaging modalities for detection Splenectomy was indicated for symptoms, hypersplenism, prophylaxis for splenic rupture and neoplastic exclusion. Intraoperatively, comprehensive exploration should be conducted to exclude the accessory spleen, which may also suffer from sarcoidosis. 


\section{References}

1. Warshauer DM and Lee JK: Imaging manifestations of abdominal sarcoidosis. AJR Am J Roentgenol 182: 15-28, 2004.

2. Jundson MA: Hepatic, splenic and gastrointestinal involvement with sarcoidosis. Semin Respir Crit Care Med 23: 529-541, 2002.

3. Kataoka M, Nakata Y, Hiramatsu J, Okazaki K, Fujimori Y, Ueno Y, Tanimoto Y, Kanehiro A, Tada S and Harada M: Hepatic and splenic sarcoidosis evaluated by multiple imaging modalities. Intern Med 37: 449-453, 1998.

4. Vagal AS, Shipley R and Meyer CA: Radiological manifestations of sarcoidosis. Clin Dermatol 25: 312-325, 2007.

5. Pérez-Grueso MJ, Repiso A, Gómez R, Gonzalez C, de Artaza T, Valle J, García A and Carrobles JM: Splenic focal lesions as manifestation of sarcoidosis: Characterization with contrast-enhanced sonography. J Clin Ultrasound 35: 405-408, 2007.

6. Warshauer DM: Splenic sarcoidosis. Semin Ultrasound CT MR 28: $21-27,2007$

7. Unver Dogan N, Uysal II, Demirci S, Dogan KH and Kolcu G: Accessory spleens at autopsy. Clin Anat 24: 757-762, 2011.

8. Thompson JC: The stomach and duodenum. In: Textbook of Surgery: The Biological Basis of Modern Surgical Practice. Sabiston DC (ed.). WB Saunders Company, Philadephia, PA, pp810-853, 1986 .

9. Soutto MM, Tempes BC, Lambert BF, Trindade EN and Trindade MR: Laparoscopic splenectomy for isolated splenic sarcoidosis. JSLS 18: 155-159, 2014.

10. Bauones S, Le Corroller T, Durieux O, Guenoun D, Del Grande J, Pirro $\mathrm{N}$ and Champsaur P: Splenic sarcoidosis mimicking neoplastic disease. J Clin Ultrasound 42: 38-41, 2014.

11. Joglekar SP, Hudson RL, Lgasundaram R and Pereira JH: 'Surgical cure' for non parathyroid hypercalcemia. World J Surg Oncol 7: 23 , 2009.

12. Zia H, Zemon H and Brody F: Laparoscopic splenectomy for isolated sarcoidosis of the spleen. J Laparoendosc Adv Surg Tech A 15: 160-162, 2005.

13. Palade R, Voiculescu1 D, Suliman E and Simion G: Splenic sarcoidosis - a case report. Chirurgia (Bucur) 107: 670-674, 2012.

14. Salazar A, Mañá J, Corbella X, Albareda JM and Pujol R: Splenomegaly in sarcoidosis: A report of 16 cases. Sarcoidosis 12: 131-134, 1995.

15. Madaule S, Lauque D, Sailler L, Arlet P and Carles P: Splenomegaly in sarcoidosis: Clinical features and outcome Analysis of 17 cases. Rev Med Interne 25: 348-356, 2004 (In French).

16. Giovinale M, Fonnesu C, Soriano A, Cerquaglia C, Curigliano V, Verrecchia E, De Socio G, Gasbarrini G and Manna R: Atypical sarcoidosis: Case reports and review of the literature. Eur Rev Med Pharmacol Sci 13 (Suppl 1): S37-S44, 2009.

17. Chen MY, Cai JT, Du Q and Wang LJ: Sarcoidosis of spleen presenting with solitary thrombopenia. Eur J of Intern Med 20: e12, 2009.

18. Ogiwara Y,MoriS, Iwama M,Sawabe M, Takemoto M,Kanazawa N, Furuta K, Fukuda I, Kondo Y, Kimbara Y, et al: Hypoglycemia due to ectopic secretion of insulin-like growth factor-I in a patient with an isolated sarcoidosis of the spleen. Endocr J 57: 325-330, 2010.
19. Cuilliere-Dartigues P, Meyohas MC, Balladur P, Gorin NC and Coppo P: Splenic sarcoidosis: An unusual aetiology of agranulocytosis. Am J Hematol 85: 891, 2010.

20. DennisBA,JajoskyRPandHarperRJ:Splenic sarcoidosis without focal nodularity: A case of 1,25-dihydroxyvitamin D-mediated hypercalcemia localized with FDG PET/CT. Endocr Pract 20: e29-e33, 2014.

21. Xiao GQ,Zinberg JM and Unger PD: Asymptomatic sarcoidosis presenting as massive splenomegaly. Am J Med 113: 698-699, 2002.

22. Fordice J, Katras T, Jackson RE, Cagle PT, Jackson D, Zaleski H and Asimacopoulos PJ: Massive splenomegaly in sarcoidosis. South Med J 85: 775-778, 1992.

23. Patel I, Ismaji M and Steuer A: Sarcoidosis presenting as massive splenic infarction. Case Rep Rheumatol 2012: 834758, 2012.

24. Bergoin C, Lamblin C and Wallaert B: Biological manifestations of sarcoidosis. Ann Med Interne (Paris) 152: 34-38, 2001 (In French).

25. Baudin B: Angiotensin I-converting enzyme (ACE) for sarcoidosis diagnosis. Pathol Biol (Paris) 53: 183-188, 2005 (In French).

26. Folz SJ, Johnson CD and Swensen SJ: Abdominal manifestations of sarcoidosis in CT studies. J Comput Assist Tomogr 19: 573-579, 1995.

27. Fiorention F, Bribani A and Rosselli A: A rare cause of the left upper quadrant abdominal pain. Intern Emerg Med 6: 55-57, 2011.

28. Kessler A, Mitchell DG, Israel HL and Goldberg BB: Hepatic and splenic sarcoidosis: Ultrasound and MR imaging. Abdom Imaging 18: 159-163, 1993.

29. Grzelak P, Augsburg L, Majos A, Stefanczyk L, Gorski P, Piotrowski W and Antczak A: Use of contrast-enhanced ultrasonography in hepatosplenic sarcoidosis: Report of 2 cases. Pol J Radiol 77: 60-63, 2012.

30. Stang A, Keles H, Hentschke S, von Seydewitz CU, Dahlke J, Malzfeldt E and Braumann D: Differentiation of benign from malignant focal splenic lesions using sulfur hexafluoride-fillled microbubble contrast-enhanced pulse-inversion sonography. AJR Am J Roentgenol 193: 709-721, 2009.

31. Tana C, Iannetti G, Mezzetti A and Schiavone C: Splenic sarcoidosis remains a diagnostic challenge. J Clin Ultrasound 42: 156, 2014.

32. Franquet T, Oteo JA, Cozcoluella R and Casas JM: Multinodular splenic sarcoidosis: Discordant CT and sonographic findings. AJR Am J Roentgenol 156: 1113-1114, 1991.

33. Raber EL, Haba J and Beck P: Splenic sarcoidosis: A case report and review of the imaging findings of multiple incidental splenic lesios as the initial presentation of sarcoidosis. Can J Gastroenterol 25: 477-478, 2011

34. Kataoka M, Nakata Y, Hiramatsu J, Okazaki K, Fujimori Y, Ueno Y, Tanimoto Y, Kanehiro A, Tada S and Harada M: Hepatic and splenic sarcoidosis evaluated by multiple imaging modalities. Internal Med 37: 449-453, 1998.

35. Sharma OP, Vucinic V and James DG: Splenectomy in sarcoidosis: Indications, complications and long-term followup. Sarcoidosis Vasc Diffuse Lung Dis 19: 66-70, 2002. 